\title{
Wie lässt sich die KVT augmentieren?
}

Bei Angsterkrankungen ist die kognitive Verhaltenstherapie (KVT) erste Wahl, als pharmakotherapeutische Alternative gelten in erster Linie SSRI. Eine KVT ergänzt durch SSRI führt oft nicht zu einer besseren Wirksamkeit der KVT, so sucht man schon lange nach weiteren Methoden zur Augmentation der KVT.

E ine vielversprechende Strategie zur - pharmakologischen Augmentation einer KVT ist die Hinzugabe des partiellen N-Methyl-D-Aspartat(NMDA)-Antagonisten D-Cycloserin (DCS), der (in Tierversuchen gezeigt) die Angstextinktion erleichtert und das erneute Aufkommen von Angst verhindert, wenn die Substanz kurz vor oder nach dem Extinktionstraining gegeben wurde. Die vorliegenden klinischen Studien sind widersprüchlich, deshalb führten MataixCols et al. eine Metaanalyse durch. Hierzu wurden 21 doppelblinde, randomisierte Studien, die 1.047 Patienten mit spezifischen Phobien, sozialer Angststörung, Panikstörung (mit/ohne Agoraphobie), Zwangsstörungen oder PTBS einschlossen, herangezogen. Die Rohdaten wurden von den Autoren erfragt und dann zusammenfassend ausgewertet. Die additive Wirkung von DCS war relativ klein, aber statistisch signifikant (Abb. 1). Die Autoren werten in der Diskussion die erzielten Ergebnisse kritisch und weisen insbesondere darauf hin, dass der Effekt der zusätzlichen Gabe von DCS nur 4 Punkte auf einer Skala von 0 bis 100 betrage, und kaum beim einzelnen Patienten unter klinischen Bedingungen feststellbar ist, was sich auch an der kleinen Effektstärke von 0,25 zeige. Zudem nahm von allen untersuchten konfundierenden Variablen ausschließlich das Publikationsjahr einen signifikanten Einfluss, in dem Sinne, dass neuere Publikationen geringere oder keine Effekte der DCS-Augmentation zeigten. Auch hatten weder Zeitpunkt noch Dosis der DCS-Gabe (meist $50 \mathrm{mg}$, eine Stunde vor der Expositionstherapie) Einfluss auf den Effekt.

\section{Kommentar}

Der augmentative Effekt einer DCS-Gabe bei einer Expositionsbehandlung ist also gering. Es erstaunt, dass mit keinem Wort auf die Nebenwirkungen bei DCS-Gabe eingegangen wird. Dies wäre aber bei einem so geringen Effekt gerade wichtig, um abschätzen zu können, ob in Zukunft DCS in dieser Indikation überhaupt weiter verwendet werden sollte (Nutzen-Risiko-Analyse). Zudem wurden hier unterschiedliche Angsterkrankungen zusammen ausgewertet, was stillschweigend die Vorannahme einschließt, dass die KVT-Prozesse bei diesen gleich sind.

Prof. Dr. med. Hans-Peter Volz

Mataix-Cols D et al. DCS augmentation of exposure-based cognitive behavior therapy for anxiety, obsessive-compulsive, and posttraumatic stress disorders. JAMA Psychiatry 2017; 74: 501-10
Hier steht eine Anzeige. Springer

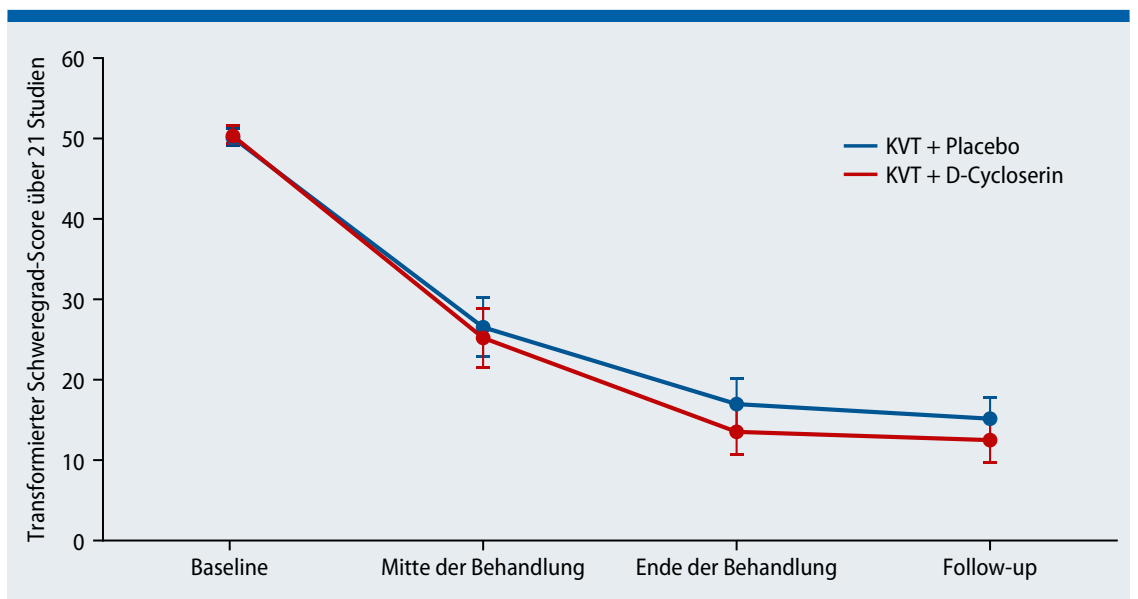

Abb. 1: Augmentativer Effekt einer DCS-Gabe im Rahmen einer Expositionsbehandlung. 